\title{
Aggression, fast and slow: Intuition also favors defensive aggression
}

\author{
Jim A.C. Everett ${ }^{1}$, Zachary Ingbretsen ${ }^{2}$, Fiery Cushman², and Mina Cikara²
}

\author{
'Department of Psychology, Leiden University \\ Department of Psychology, Harvard University
}

This pre-print is dated December $11^{\text {t" }} 2018$ and is currently undergoing peer review. Please do not cite without permission from the authors.

\begin{abstract}
Author Note
The authors gratefully acknowledge support from the National Science Framework (1551559) awarded to MC; the US-UK Fulbright Commission and the European Union's Horizon 2020 research and innovation programme under the Marie Skłodowska-Curie grant (707404) for JACE.
\end{abstract}

\section{Corresponding Author:}

Dr. Jim A.C. Everett (Jim.ac.everett@gmail.com)

Pieter de la Court Building, Wassenaarseweg 52

2333 AK Leiden, Netherlands 


\begin{abstract}
In certain threatening social encounters, humans seem to act distrustfully, uncooperatively, even aggressively by default, and must reason themselves towards comity and moderation. Yet, the contemporary scientific literature mostly supports the opposite view, according to which we are "intuitively prosocial": acting cooperatively by default in positive-sum interactions in games such as the Prisoner's dilemma. Could a change in the nature of the game elicit a different profile of default behavior? Across four studies we investigate whether intuition might also favor defensive aggression. We develop a "preemptive strike game" in which two players make a series of decisions about whether to liveand-let-live, or instead pay a small cost to imposing a large cost on the other player, knocking them out of the game. In this setting we find that default aggression prevails. Moreover, this aggressive tendency can be overridden when playing the game against in-group members, but tends not to be when playing against out-group members. In short, when faced with a social partner who may choose to harm them, people default towards defensive aggression.
\end{abstract}

Keywords: pre-emptive striking; aggression; dual process; intuition; social heuristics 


\section{Aggression, fast and slow: Intuition also favors defensive aggression}

A growing body of experimental research suggests that humans are cooperative by default, arising from "processes that are intuitive, reflexive, and even automatic" (Zaki \& Mitchell, 2013, p. 466). Time pressure makes us more cooperative (e.g. Rand et al 2014), as does conceptual priming of intuition (e.g. Lotz, 2015), and cooperative people self-report relying on non-deliberative processes (Levine, Barasch, Rand, Berman, \& Small, 2018). Neuroimaging data further suggest cooperation holds "intrinsic" value (e.g. Dawes et al., 2012; Tricomi, Rangel, Camerer, \& O’Doherty, 2010; Zaki, López, \& Mitchell, 2014; Zaki \& Mitchell, 2011). Conversely, people are deeply averse to harming others-sometimes more so than they are for themselves (e.g. Crockett, Kurth-Nelson, Siegel, Dayan, \& Dolan, 2014), and sometimes to the point of being reluctant to even simulate harmful actions (e.g. Cushman, Gray, Gaffey, \& Mendes, 2012). The cognitive basis of this aversion is often unavailable to introspection, however (Cushman, Young, \& Hauser, 2006), suggesting that it may originate with automatic and intuitive processes (Greene, 2008).

The conclusion that humans are intuitive cooperators is hard to reconcile, however, with the observation that humans are also sometimes distrustful, uncooperative, or even aggressive by default - especially when faced with threat. Consistent with this possibility, many studies highlight the role that automatic processes play in defensive aggression (De Dreu, Scholte, van Winden, \& Ridderinkhof, 2015; Nelson \& Trainor, 2007) and intergroup bias (e.g. Cikara \& Van Bavel, 2014; Dunham, Baron, \& Banaji, 2008). How might we begin to develop a principled understanding of the circumstances under which default cooperation versus default aggression prevail?

We approach this problem by investigating the default response to a kind of social dilemma that, while commonplace in the real world, is remarkably understudied in the lab: the decision of whether to engage in pre-emptive strikes (e.g. Böhm, Rusch, \& Gürerk, 2016; 
Halevy, 2017; Mifune, Hizen, Kamijo, \& Okano, 2016; Simunovic, Mifune, \& Yamagishi, 2013). In our "pre-emptive strike game" two players make a series of decisions about whether to live-and-let-live, or whether to pay a small cost to impose a large cost on the other player and thereby knock them out of the game. We test whether default aggression prevails in the pre-emptive strike game, in contrast to the large body of results showing default cooperation in social dilemmas modelled on the prisoner's dilemma. We also test whether this aggressive tendency can be overridden when playing the game against in-group versus out-group members. Our goal is to clarify whether default aggression exists alongside default cooperation, and provide a basic map of where each should be expected.

\section{From Intuitive Cooperation to Intuitive Aggression}

While previous work has often emphasized the role of intuitive cooperation and deliberative selfishness (e.g. Rand, 2016; Rand, Greene, \& Nowak, 2012), there also seems to be an important role for more intuitive processes in aggression (e.g. De Dreu et al., 2015; Nelson \& Trainor, 2007; Sanfey, Rilling, Aronson, Nystrom, \& Cohen, 2003). Aggression researchers tend to distinguish between more controlled, purposeful, and goal-oriented instrumental aggression that is regulated by higher cortical brain systems, and more impulsive or intuitive reactive, or defensive aggression that is more dependent on the hypothalamic and limbic systems (Nelson \& Trainor, 2007). For example, in a predator-prey contest game, defensive aggression appears to depend on sub-cortical circuitries associated with intuitive responding (like the amygdala), whereas aggression geared at subordinating others, and appropriating other's resources is typically more controlled and calculated (De Dreu et al., 2015).

Building on this insight, we investigate a classic setting that elicits defensive aggression: pre-emptive strikes. The basic logic of a pre-emptive strike is to attack another agent in order to gain a strategic advantage, and to do so before the other agent can attack 
you. In their classic essay, Schelling (1960) uses the example of an armed homeowner who is faced with a burglar, also with a gun. If the homeowner believes that the burglar might shoot, then the best response is for her to shoot first in a surprise attack and incapacitate the burglar before he can hurt her. But suspecting this might happen, the burglar might also be tempted to shoot the homeowner first to incapacitate her before she can hurt him. In this way, both sides can end up attacking, even though neither had any desire to hurt the other.

Modelling such situations in experimental games, research has confirmed that preemptive strikes are driven by fear of attack by another (for this reason they have also been referred to as cases of "fear-based aggression": Simunovic, Mifune, \& Yamagishi, 2013). In particular, people launch pre-emptive strikes against others who they fear may be motivated to the harm them out of spite (Abbink \& de Haan, 2014, p. 195). Such decisions arise rapidly, often within a few seconds (Simunovic et al., 2013), consistent with the possibility that they represent the default, intuitive response (though the game structure in previous work has necessarily induced time pressure, so there was no deliberation comparison group). Defensive aggression seems to rely on more reactive, emotional brain processes (e.g. De Dreu et al., 2015; Nelson \& Trainor, 2007) and occurs very rapidly (Simunovic et al., 2013), but no work has directly looked at the effect of manipulating a reliance on intuition through induced time pressure on defensive aggression. Therefore, we provide a causal test of the relationship between decision-time and defensive aggression by manipulating time pressure in the context of a pre-emptive strike game tapping defensive aggression.

\section{Aggression Towards Out-groups}

Pre-emptive strikes can occur in all areas of social life but, as any student of politics and history could attest, such strikes seem especially likely when groups perceive that they are under existential threat. Following the 9/11 terrorist attacks, for example, George Bush justified a pre-emptive in the Middle East in order to prevent future attacks, arguing that 
Americans must "be ready for preemptive action when necessary to defend our liberty and to defend our lives" (X; our italics). Similar psychological processes relating to pre-emptive strikes might help explain the racial disparity in police and security gun shootings. In 2012, George Zimmerman - an armed member of the local community watch - killed 17-year old African-American teenager Trayvon Martin, claiming self-defense. Zimmerman had a firearm while Martin was "armed" only with a bag of Skittles and ice tea, but the fear that Martin could harm Zimmerman led him to quickly reach for the gun and fire the fatal shots. Dangerous situations in which there is a perceived threat to safety are likely to engender preemptive strikes, and this is likely to become especially pronounced when the situation involves interactions between people from groups in conflict.

In social situations generally, "the simple fear of nastiness is sufficient to make a large majority inflict considerable damage on another person" (Abbink \& de Haan, 2014, p. 195). When it comes to intergroup contexts this fear of nastiness is likely to be heightened, with much prior research showing that people tend to fear and distrust out-group members more than in-group members (e.g. Chang, Krosch, \& Cikara, 2016; Cottrell \& Neuberg, 2005; Insko \& Schopler, 1998; Pemberton, Insko, \& Schopler, 1996; Riek, Mania, \& Gaertner, 2006; Sherif, 1966; Stephan, Renfro, \& Davis, 2008). In fact, cynical leaders may even exploit this fear in order to encourage collective aggression against out-groups (De Dreu \& Gross, Forthcoming, p. 36) and support for punitive immigration policies (Martinez, Feldman, Feldman, \& Cikara, Under Review). We have good reasons, therefore, to expect higher levels of pre-emptive striking against out-group members due to fear and distrust.

In previous work we investigated a related question, asking whether people exhibit intuitive cooperation, or instead intuitive defection, against out-group members in the traditional Prisoner's Dilemma setting (Everett, Ingbretsen, Cushman, \& Cikara, 2017). Consistent with prior work, we found that time pressure made people more 
cooperative overall. Moreover, the magnitude of this effect was not detectably different for in-group versus out-group members. (Consistent with the large literature on intergroup bias, we also found that people were more cooperative with in-group members than with out-group members).

Here, we take a parallel approach in the context of pre-emptive strikes. We anticipated that our pre-emptive strike game (PSG) game would prompt intuitive aggression (in contrast to the PDG), and that it would prompt heightened aggression towards out-group members (consistent with the PDG). Moreover, our experimental design would reveal any interaction between these forces (which was not observed for the PDG). For instance, we may observe comparable default aggression to both in-group and out-group members, but a deliberative "correction" to selectively reduce aggression towards in-groups. Or, we may observe extreme default aggression selectively towards outgroup members, but a deliberative "correction" that eliminates this bias between groups.

\section{Present Research}

In this paper we report four experiments investigating whether in a novel economic game 1) participants under time pressure exhibit greater defense-based aggression in general, and 2) whether this effect is moderated by the group membership of participants' interaction partner. To test this, we used a new game - the pre-emptive strike game (PSG). Adapted from previous work by Simunovic and colleagues (2013), our PSG allowed us to implement time pressure in order to facilitate a reliance on intuition. The PSG (see Fig. 1) has 5 rounds in which, in each round, participants choose between two options: "grey" and "red." The grey option does nothing; if both players choose grey, both players to proceed to the next round. If the players reach the 5th and final round, they both get a payoff of 1500 points (which is then converted into money). On each round, however, players have the option to choose "red". This inflicts a small cost of 100 points on the chooser's final payoff (i.e. they end with 1400), 
but a much greater cost of 1000 points on her partner (i.e. they end with 500). Also, it causes the entire game to finish, thus preventing the opponent from inflicting any retaliatory cost. If a player chooses the red option, then, it constitutes a pre-emptive strike: a severe attack that imposes a large harm at a smaller cost and terminates the interaction. In the PSG, participants play sequentially, alternating the decision order with one player moving first on the first round, then the second player moving first on the second round, and so on.

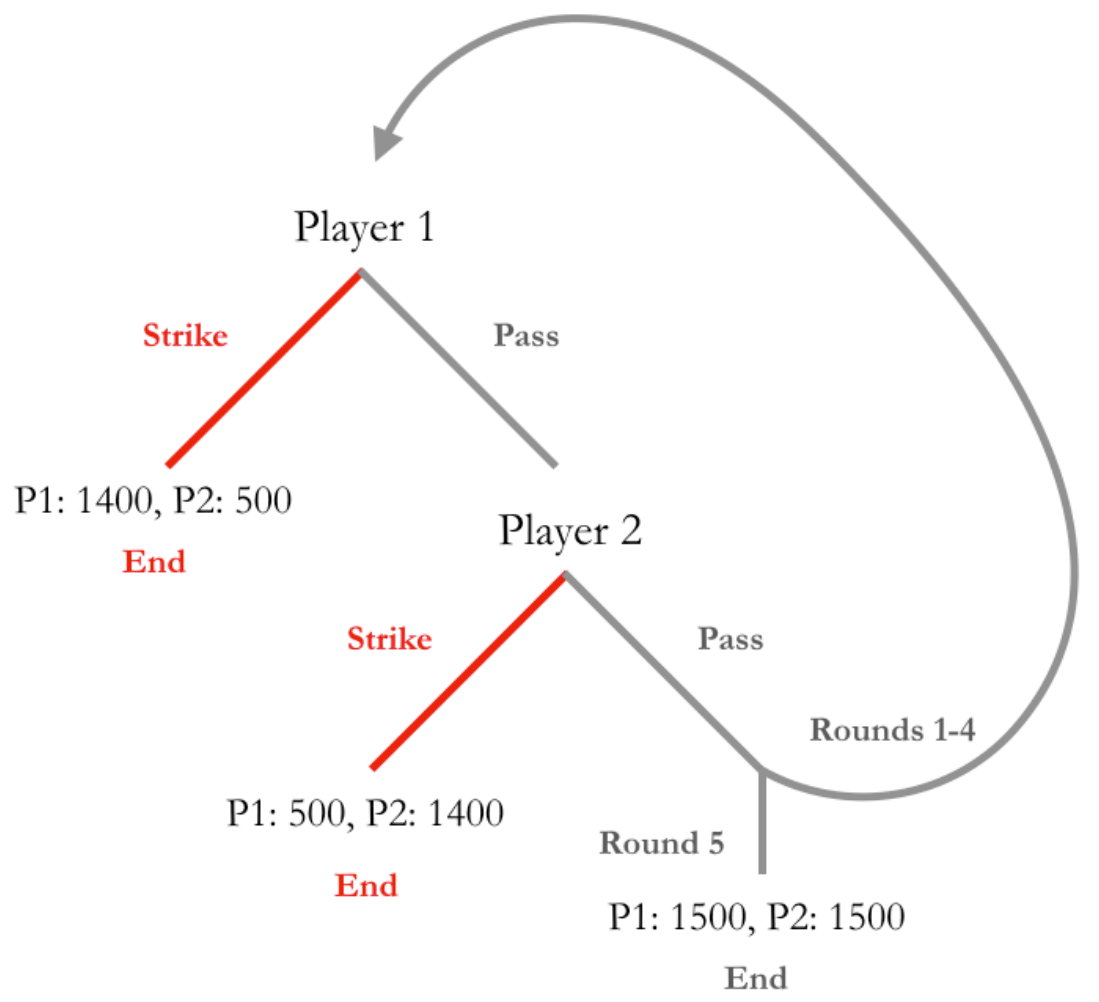

Figure 1. Our pre-emptive strike game (PSG) has 5 rounds in which, in each round, participants choose between two options: "grey" and "red." The grey option does nothing; if both players choose grey, both players to proceed to the next round. If the players reach the 5th and final round, they both get a payoff of 1500 points (which is then converted into money). On each round, however, players have the option to choose "red". This inflicts a small cost of 100 points on the chooser's final payoff (i.e. they end with 1400), but a much greater cost of 1000 points on her partner (i.e. they end with 500). The red option causes the entire game to finish, thus preventing the opponent from inflicting any retaliatory cost. 
It is important to emphasize the key differences between the PSG and the one-shot Public Goods Dilemma (PDG), which has typically been the focus of work on time pressure effects in cooperation. Famously, in the one-shot PDG, the pure Nash equilibria is for defection: a player maximizes her payoff by defecting, irrespective of her partner's move. Purely self-interested game play will not, therefore, depend on trust of the opponent. Like the Stag Hunt game, however, the PSG differs strongly in this regard. If you are certain that your partner will cooperate, then you are strictly worse off by paying a cost to strike preemptively - there is no point hitting a person who you trust, and so mutual cooperation is a payoff-dominant Nash equilibrium. On the other hand, if you do distrust a person, then engaging in a first strike can be rational. In this way, pre-emptively striking constitutes a riskdominant Nash equilibrium because if uncertainty exists about the other player's action, striking will provide a higher expected payoff (Harsanyi \& Selten, 1988). This strong dependency on trust is why the PSG (rather than the PDG) may be especially suited to studying the intersection of automatic fear and intuitive pro-sociality.

In all four experiments, we had participants play the PSG either 1) under time pressure or with no time constraints, and 2) with a political in-group or out-group member. In these studies participants always played as the first mover for the first round; the second player was computer-generated and always chose the grey option (because if the fictitious second player chose red, the game would end). Note that while the implementation varied slightly across the experiments (i.e., whether participants made decisions using the mouse (Studies 1-2) or a keyboard press (Studies 3-4); or whether group membership was indicated with text (Studies 1) or a graphic (Studies 2-4)), the core game did not change.

\section{General Methods}

Our four experiments all followed an identical general template. 


\section{Open Science}

For all the experiments we report in this manuscript, we report all measures, manipulations, and exclusions. All data, analysis code, and experimental materials across all four experiments are available for download at: https://osf.io/7uk3c/?view only=c1854a61393044529b712492b7becd90. Our design, analysis plan, and hypotheses for Experiment 4 were pre-registered:

https://osf.io/dquxp/?view only=c691681e8c1f4874a2c2cde055c6d82d

\section{Participants}

American participants completed the survey via MTurk and were paid $\$ 0.60$ for their time, with the potential to earn an additional bonus depending on their decisions in the game.

\section{Design}

We used a between-subjects 2 (Time Manipulation: Time Pressure vs. Control) x 2 (Group: In-group vs. Out-group) design, such that participants played the PSG with either a political party in-group or out-group member, and either had to make their decisions in the game under time pressure or with no time instructions given. In Experiment 4 we also included an extra "neutral" condition that did not provide information about the ingroup or outgroup status of the other player.

\section{Procedure}

All experiments were implemented online via Qualtrics. Upon beginning the experiment, participants were told that they would be playing a game with another MTurker that gave bonus points that, at the end of the game, would be translated into real money. Participants were given details about the structure of the PSG, including the fact that they would always be moving first in the first round (and then the partner would move first in the second round, and so on), and required to answer four comprehension questions to ensure they had understood the game (see materials on OSF for full wording). To ensure 
comprehension, participants could only move forward once they had answered these correctly.

After successfully answering these comprehension questions, participants were told that they would be playing in teams based on political party and that their partner could be from the same team (in-group) a different team (out-group). To make salient these group identifications, after indicating which party they themselves identify with, they answered three questions assessing their feelings about both parties ("I value / like / feel connected to the Democrat [Republican] party"). Participants who indicated that they were political independents were randomly assigned to either team, and their data was subsequently excluded during analysis.

Participants were told that prior to making their decision, they would be told the group membership of their partner, and to ensure participants paid attention to this, were told that they would receive an additional $\$ 0.05$ bonus if they remembered this correctly at the end of the experiment. In Experiment 1, group membership was indicated to participants with text indicating that, for example, "You are playing with someone from THE SAME [OPPOSING] team... Your partner is a DEMOCRAT [REPUBLICAN]”. In Experiments 2,3 and 4 we replaced the text indicating group membership with card images, and made it clear to participants that their own group membership would be known by the other player.

On the subsequent page, participants began the game and made their decision. Participants in the control condition were given no instructions about timing and had as long as they needed to make the decision. Participants in the intuition condition were instructed they had to make their decision within 3 seconds. A count-down clock indicated the remaining time, and the page automatically moved on after 4 seconds. Responses were recorded by mouse click in Experiments 1 and 2, and by keyboard press in Experiments 3 and 
4. If participants did not make a decision in the allocated time, they were directed to the end of the survey and debriefed. If participants chose 'red', the game immediately ended and the participant earned 1400 points and the other player 500 points. If the participant chose 'grey', they proceeded to the next round, and if they continued to choose grey they would reach the fifth and final round and both players would receive 1500 points. While we were interested in the decisions on the first round, we needed to have the appearance of multiple rounds with sequential turn-taking because a simultaneous decision would have induced time pressure in a rush to answer first, as in Simunovic et al. (2013).(Specifically, we needed at least three rounds. We needed more than one round because then there is no fear of attack for Player 1 , and we needed more than two rounds because then there is no fear of attack for Player 2. We told participants they could randomly be assigned to play as either Player 1 or Player 2, and this would determine who moves first on the first round, then first on the second round and so on. If there are only two rounds, Player 2 never has a motive to attack because the game ends after their decision anyway and so there is no fear of retaliation. To ensure equal incentives, we therefore needed at least three rounds so that both players would have a fear of future attack when they made their decision. We chose to implement five rounds as a 'nice' number that would give both players two chances to make their decisions, while also not being unnecessarily long). Once the game was complete, participants provided simple demographic information and reported the group membership of their partner (to serve as a comprehension check).

The procedure did vary slightly based on whether participants were in the intuition or control condition. Before receiving information about the main PSG, participants in the intuition condition received three practice questions to familiarize them with what 3 seconds felt like. In Experiments 1 and 2, these consisted of three simple multiple-choice questions (e.g. "What country is Paris in?”: a) France, b) Germany, c) Italy) that participants were 
required to answer in less than 3 seconds. In Experiments 3-4 these consisted of three true or false statements, which participants answered using a keyboard press. To ensure that participants did not systematically differ in their ability to make quick decisions, after completing the main game participants in the control condition also received the three timepressure practice questions and completed a bonus round under time pressure. This allowed us to avoid a selection bias confound.

\section{Experiment 1}

In Experiment 1 we sought to explore pre-emptive striking in a new, revised game that allowed us to manipulate whether participants faced time pressure or not, playing with either a political party in-group or out-group member.

\section{Method}

Among 1104 participants, we excluded those who completed the survey more than once $(N=107)$, were political independents $(N=153)$, failed to make a decision in the time pressure condition $(N=67)$, or failed to complete the time pressure bonus round in the control condition $(N=13)$, and/or failed a simple comprehension check about the group membership of the person they played with $(N=13)$. This left a final sample of 751 participants (380 female, $\left.M_{\mathrm{agc}}=33.58, S D_{\mathrm{agc}}=10.81\right)$.

\section{Results}

We conducted a logistic regression predicting responses on only the first round of the game, including both the main effects of time manipulation and partner group as well as the interaction term (setting the in-group as the reference category for the group factor and the control condition as the reference category for the time manipulation factor). We observed main effects of both group, $B=0.80, S E=0.27, Z=2.93, p=0.003$, and time pressure manipulation, $B=0.59, S E=0.29, Z=2.05, p=0.040$, qualified by a significant interaction between group condition and time manipulation, $B=-0.81, S E=0.39, Z=-2.08, p=0.038$. 
Breaking this down, we found that under time pressure participants were equally likely to attack an in-group (19\%) or out-group member (19\%), $B=0.01, S E=0.28, Z=0.02, p=$ 1.00. In contrast, in the control conditions where participants had the chance to deliberate, they were significantly less likely to attack an in-group member (12\%) than an out-group member (23\%), $B=-0.80, S E=0.27, Z=-2.93, p=.018$. Overall, then, our results show that when given the potential to deliberate, participants were significantly less likely to defect against an in-group, but not out-group member (see Figure 2).

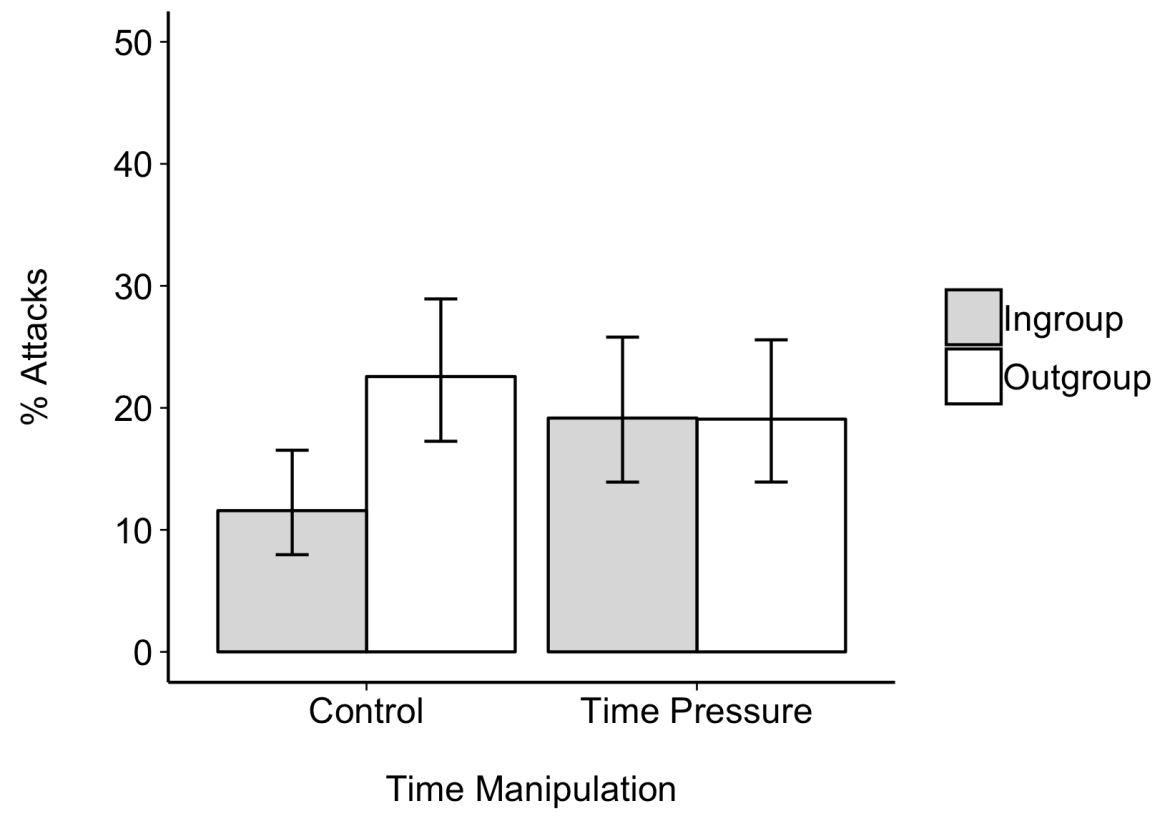

Figure 2. Under time pressure, participants were equally likely to attack an in-group or outgroup member, but when they had the chance to deliberate were significantly more likely to attack an out-group than in-group member. Error bars represent $95 \%$ confidence intervals (Experiment 1).

\section{Experiment 2}

In Experiment 2, we sought to replicate our findings in Experiment 1 while implementing some minor methodological changes. We were concerned that, in Experiment 1, participants may have been unsure whether their own group membership was known by 
their partner, which is problematic given the role of reputational concerns in in-group favoritism (Mifune, Hashimoto, \& Yamagishi, 2010). To address this, Experiment 2 used graphical representations of team membership - team 'cards' - rather than the textual descriptions used in Experiment 1. By using team cards, we could both enhance the saliency of the teams, and ensure that participants were aware that just as they would see the other player's card, their partner would see the participant's card.

\section{Method}

\section{Participants}

Among 1004 participants, we excluded those who completed the survey more than once $(N=19)$, were political independents $(N=161)$, failed to make a decision in the time pressure condition $(N=47)$, or failed to complete the time pressure bonus round in the control condition $(N=10)$, and/or failed a simple comprehension check about the group membership of the person they played with $(N=39)$. This left a final sample of 728 participants $\left(418\right.$ female, $\left.M_{\mathrm{agc}}=35.21, S D_{\mathrm{agc}}=11.73\right)$.

\section{Results}

Using the same logistic regression analysis as in Experiment 1, we failed to replicate the significant interaction between group condition and time manipulation observed before, $B$ $=-0.28, S E=0.40, Z=-0.70, p=0.485$ and, while there was a main effect of group membership, $B=0.58, S E=0.29, Z=2.01, p=0.044$, there was no main effect of time pressure manipulation, $B=0.39, S E=0.30, Z=1.29, p=0.197$. Participants were more likely to attack an out-group member (20\%) than an in-group (14\%), but this did not significantly differ between the control and time pressure conditions. While not significant, it is useful to note that the pattern of means was identical to Experiment 1: slightly larger difference in attack rates towards an in-group (12\%) than out-group (20\%) in the control 
conditions, compared to an in-group (17\%) and out-group (21\%) in the time pressure conditions (see Figure 3).

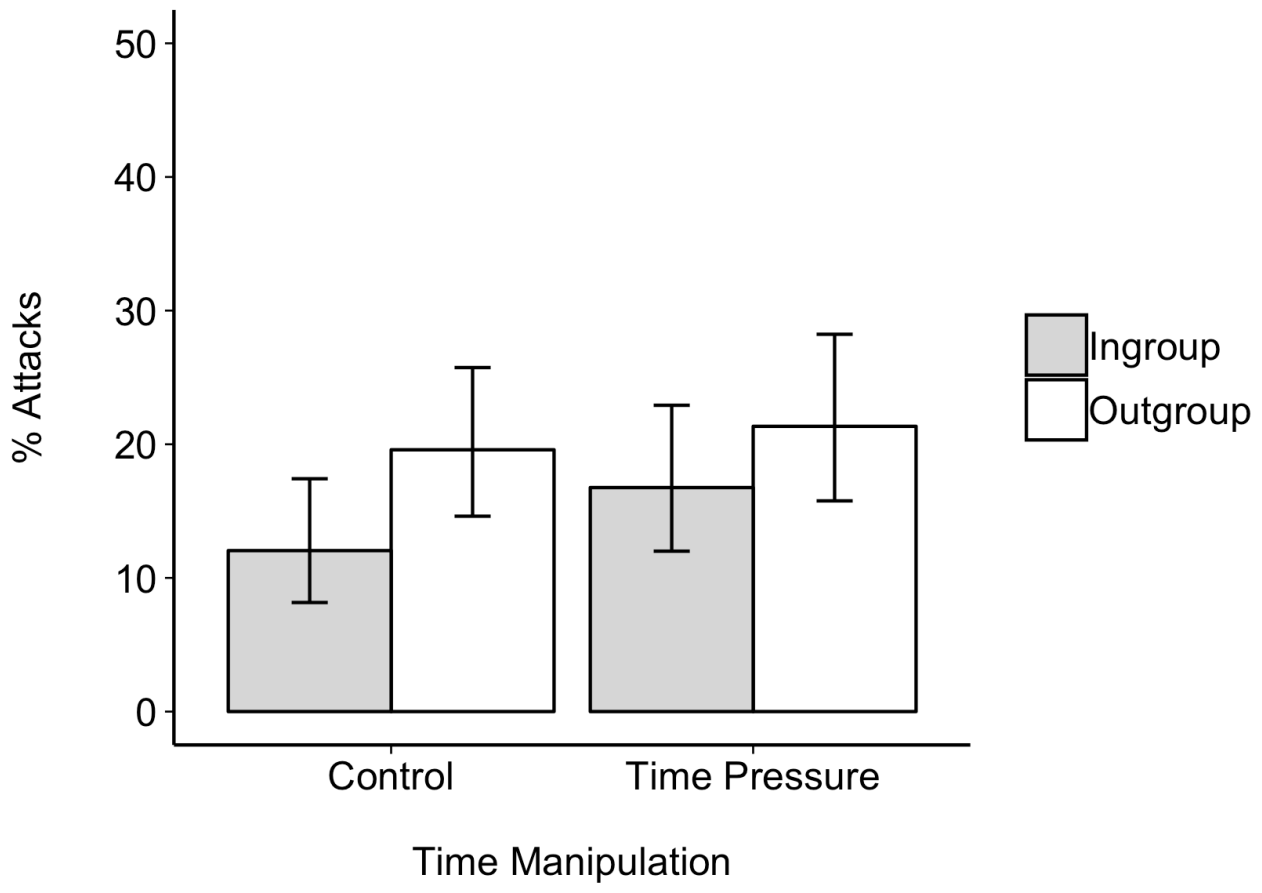

Figure 3. Participants were more likely to attack an out-group than in-group member, and while the directional pattern of results was consistent with Experiment 1, there was no significant interaction of time pressure with group membership in predicting defensive attacks. Error bars represent 95\% confidence intervals (Experiment 2).

\section{Discussion}

In Experiment 2 the directional pattern of results was consistent with Experiment 1 but several contrasts of interest were non-significant. One possible explanations was that players in the control (no time pressure) condition may have thought that they needed to make a preemptive strike decision before a freely-responding partner, thus perceiving time pressure where none really existed. We addressed this possibility in Experiment 3. 


\section{Experiment 3}

In Experiment 3, we sought to again compare aggression in the PSG towards an ingroup or out-group, under time pressure or not, but again made some changes to the implementation to address concerns with the previous studies. Most important was the concern that participants in the control condition might also be perceiving time pressure (albeit to a lesser degree than participants explicitly given the time manipulation). To address this, we made several changes. First, we emphasized to participants that they would be taking turns to make decisions, rather than deciding simultaneously. In the instructions at the beginning of the survey, we only told participants that which player would moves first on the first round would be randomly determined when assigned their partner. This allowed us to present participants with the information that they would be moving first immediately before being shown the team card of the other player and making a decision: "You will make your decision first. Because you have been assigned to go first on this first round, the other person will only be allowed to submit their response after you have submitted yours, though we will reveal your choices to each other at the same time." By highlighting that decisions were sequential, not simultaneous, we aimed to eliminate the perception that participants needed to respond quickly to 'beat' the other player to the decision. Second, we ensured that response times were recorded for the main decision round of the control condition. Third, we gave participants a manipulation check question at the end of the survey in which they were asked to indicate how rushed they felt playing the game. Aside from these changes designed to address the potential concern of participants in the control condition perceiving some time pressure, we also moved away from having participants make decisions by pointing-andclicking with their mouse, to make their decision by pressing one of two keys on the keyboard. This allowed us to have participants make even quicker decisions, and - we hoped - encourage a sense of urgency for participants in the time pressure condition. 


\section{Method}

\section{Participants}

Among 1007 participants we excluded those who completed the survey more than once $(N=12)$, were political independents $(N=145)$, failed to make a decision in the time pressure condition $(N=69)$ or failed to complete the time pressure bonus round in the control condition $(N=39)$, and/or failed a simple comprehension check about the group membership of the person they played with $(N=29)$. This left us with a final sample of 713 participants (318 female, $\left.M_{\mathrm{agc}}=34.67, S D_{\mathrm{agc}}=10.67\right)$.

\section{Manipulation Check}

To confirm that our manipulation had worked as intended, we compared how long participants took, and how rushed they felt doing so, between the time pressure and control conditions. As expected, participants took significantly longer to make their decisions in the control condition $($ mean $=5.45$, median $=3.19)$ than the time pressure condition (mean $=$ 2.16$, median $=1.81), t(388)=3.63, p<0.001$. Similarly, participants reported feeling significantly more rushed in the time pressure condition $(M=4.41, S D=1.94)$ than the control condition $(M=2.49, S D=1.72), t(660)=-13.78, p<0.001$.

\section{Results}

As in the previous studies, we conducted a logistic regression including both the main effects and interaction term, and setting the in-group as the reference category for the group factor, and the control condition as the reference category for the time condition. We observed main effects of both group membership, $B=0.93, S E=0.27, Z=3.46, p<0.001$, and time pressure manipulation, $B=1.12, S E=0.27, Z=4.11, p<0.001$, and as predicted these main effects were qualified by a significant interaction between group condition and time manipulation, $B=-1.14, S E=0.36, Z=-3.17, p=0.002$. 
Breaking this down, we found that under time pressure participants were equally likely to attack an in-group (32\%) or out-group member $(28 \%), B=0.21, S E=0.24, Z=$ $0.88, p=.816$. In contrast, when participants had the chance to deliberate they were significantly more likely to attack an out-group (28\%) than in-group member (13\%), $B=-$ $0.93, S E=0.27, Z=-3.46, p=.003$. Indeed, while time pressure made no difference to the incidence of attacking against an out-group member, $B=0.02, S E=0.24, Z=0.09, p=.999$, the possibility to deliberate led to significantly fewer attacks against an in-group member than when participants were under time pressure, $B=-1.12, S E=0.27, Z=-4.11, p<.001$ (see Figure 4).

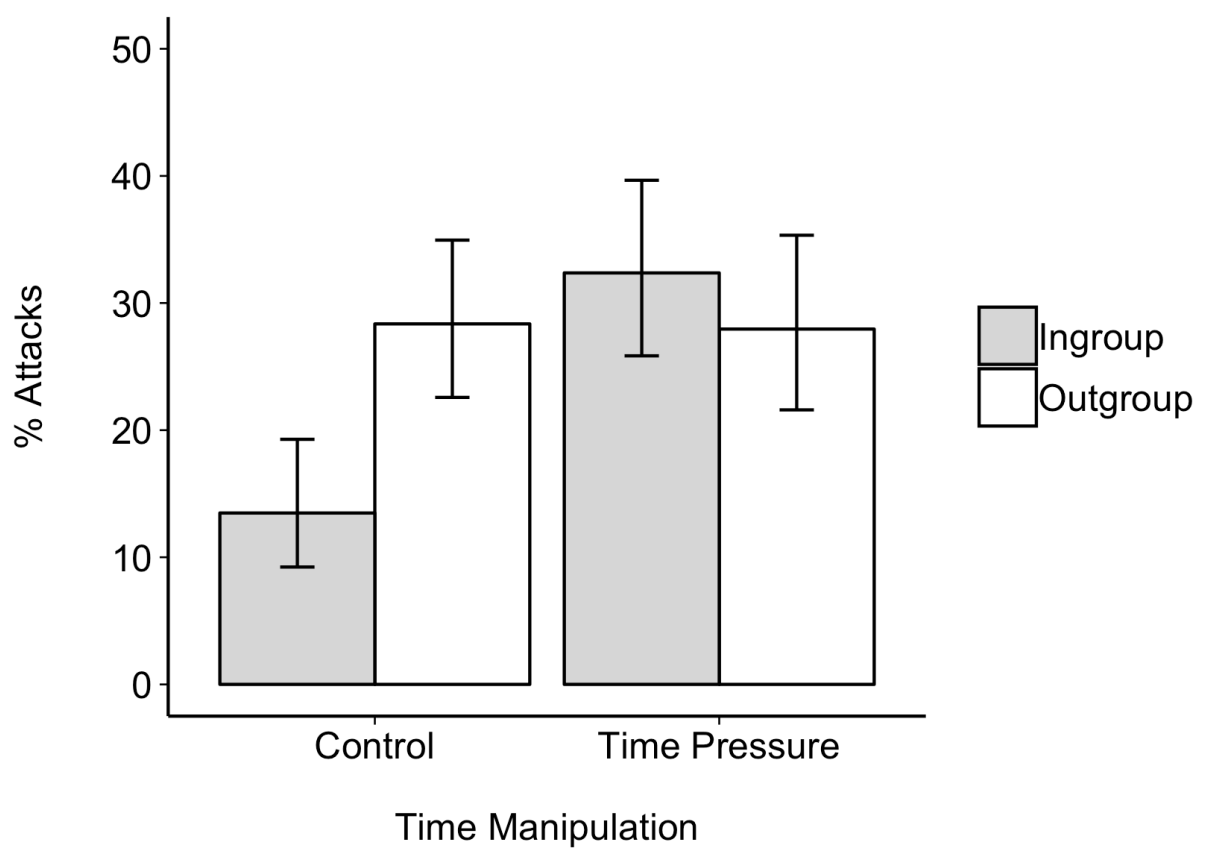

Figure 4. Under time pressure, participants were equally likely to attack an in-group or outgroup member, but when they had the chance to deliberate were significantly more likely to attack an out-group than in-group member. Error bars represent $95 \%$ confidence intervals (Experiment 3). 


\section{Discussion}

In Experiment 3 we replicated our interaction from Experiment 1, demonstrating that under time pressure, participants were equally likely to attack an in-group or out-group member, but when they had the chance to deliberate were significantly more likely to attack an out-group than in-group member.

\section{Experiment 4}

We have thus far provided mixed, but strongly suggestive, evidence that a reliance on intuition can lead to fear-motivated aggression towards both in-group and out-group members, and that the ability to deliberate can selectively reduce this aggression towards ingroup members. One question remains, however: is this driven more by fear of the out-group or love of the in-group? When given the chance to deliberate, would participants treat an unidentified other more like an out-group (suggesting that our results are driven more by ingroup love than a specific fear of the out-group), or would they treat an unidentified other more like an in-group (suggesting that our results are driven more by fear of the out-group than love of the in-group)?

\section{Method}

\section{Participants}

We recruited participants and analyzed their data in accordance with the preregistration and the previous experiments, Among 1665 participants, we excluded those who completed the survey more than once $(N=15)$, were political independents $(N=255)$, failed to make a decision in the time pressure condition $(N=58)$ or failed to complete the time pressure bonus round in the control condition $(N=31)$, and/or failed a simple comprehension check about the group membership of the person they played with $(N=101)$. This left a final sample of 1037 participants (568 female, $\left.M_{\mathrm{agc}}=35.44, S D_{\mathrm{age}}=11.30\right)$. 


\section{Design}

The design and procedure were identical to Experiment 3, with the exception of our adding in an "unknown" condition to the group manipulation. This gave us a betweensubjects 2 (Time Manipulation: Time Pressure vs. Control) x 3 (Group: In-group vs. Outgroup vs. Unidentified) design, such that participants played the PSG with either a political party in-group member, out-group member, or unidentified other, and either had to make their decisions in the game under time pressure or with no time instructions given.

\section{Manipulation Check}

To confirm that our manipulation had worked as intended, we compared how long participants took, and how rushed they felt doing so, between the time pressure and control conditions. As expected, participants took significantly longer to make their decisions in the control condition $($ mean $=6.29$, median $=2.95)$ than the time pressure condition $($ mean $=$ 2.08$, median $=2.10), t(535)=3.97, p=<0.001$. Similarly, participants reported feeling significantly more rushed in the time pressure condition $(M=4.42, S D=1.91)$ than the control condition $(M=2.49, S D=1.64), t(990)=-17.44, p=<0.001$.

\section{Results}

To analyse behaviour in the PSG, we conducted a logistic regression, including both the main effects and interaction term, and setting the in-group as the reference category for the group factor, and the control condition as the reference category for the time condition factor.

Consistent with the previous studies, we observed a significant main effect of time pressure manipulation, $B=0.91, S E=0.26, Z=3.58, p<.001$, and a main effect of attack rates towards the in-group versus the out-group, $B=0.57, S E=0.26, Z=2.18, p=.029$, but not between the in-group and unidentified others, $B=0.09, S E=0.28, Z=-0.30, p=.761$. Surprisingly, there was no interaction of the time pressure manipulation with group 
membership, and this was the case both when looking at the interaction of time pressure with in-group versus out-group, $B=-0.43, S E=0.34, Z=-.13, p=.208$, and the interaction of time pressure with the in-group versus unidentified others, $B=-0.10, S E=0.37, Z=-0.28, p$ $=.780$. Note that while there were no interaction effects of time pressure with group membership, the pattern of results was descriptively the same as before: under time pressure, participants were similarly likely to attack an out-group (38\%), an in-group (34\%), or an unidentified other (30\%). When given the chance to deliberate, however, participants did seem more likely to attack an out-group member (27\%) than an in-group (17\%) or unidentified other (16\%) (see Figure 5).

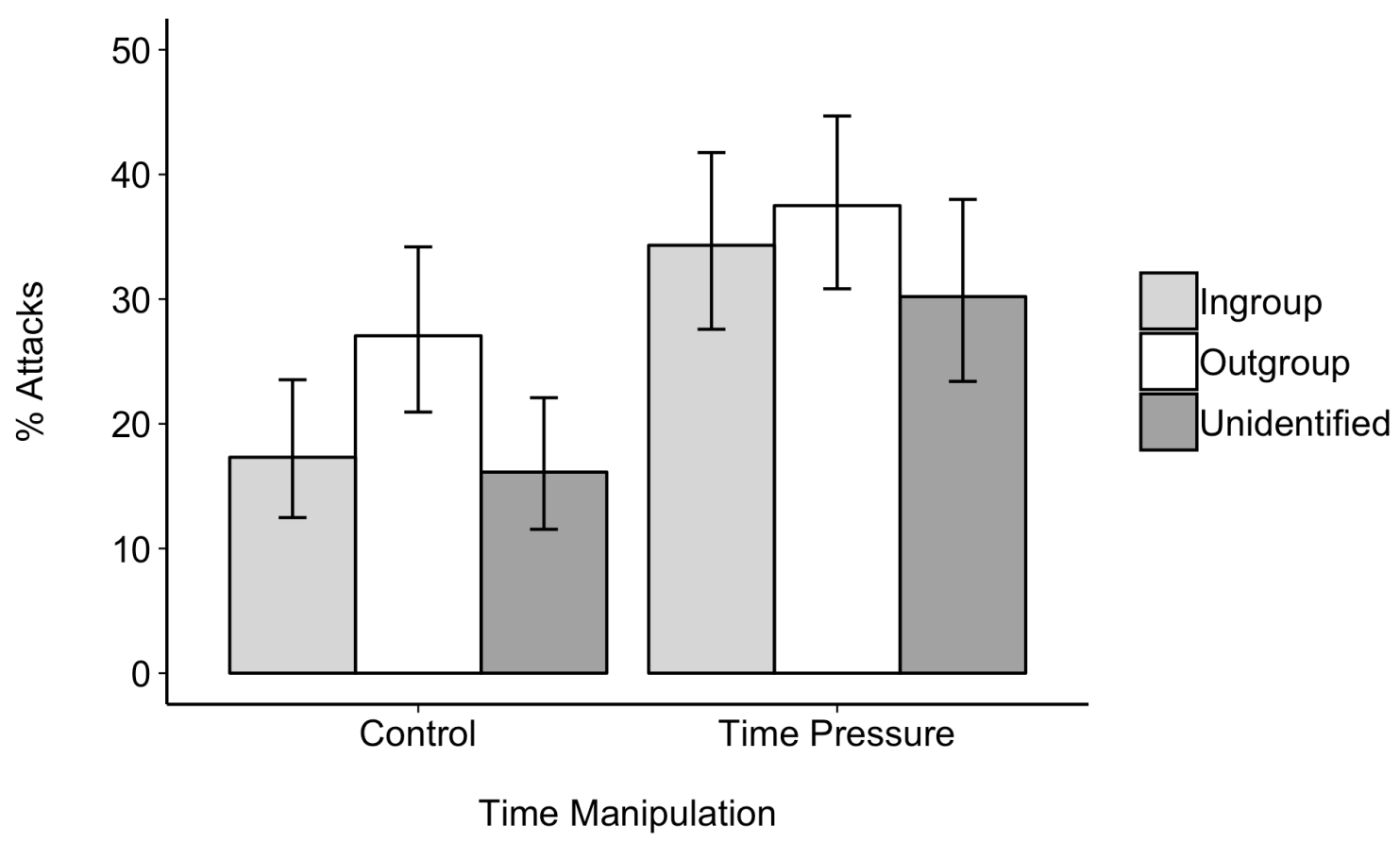

Figure 5. Under time pressure, participants were more likely to attack all partners. While we again observed the descriptive pattern of fewer attacks towards an in-group member than an out-group in the control condition, the previously-found interaction was not significant. Error bars represent 95\% confidence intervals (Experiment 4). 


\section{Discussion}

In Experiment 4 we again observed that participants were more than twice as likely to attack an out-group member over an in-group member (or unidentified other) when given the chance to deliberate, but not when making decisions under time pressure. That participants treated an unidentified other more similarly to an in-group than an out-group suggests that our results are driven by a specific fear of the out-group, rather than a generic dislike of nonin-group members. That is, if our previous findings can be explained more by in-group love, we should expect participants to attack more everyone who is not an in-group member; if our previous findings can be explained more by out-group fear, we should expect participants to attack less everyone who is not an out-group member. (Of course, it's also possible that deliberation allows for participants to decide to behave spitefully, but given all the previous work suggesting that pre-emptive strikes are driven almost entirely by fear rather than spite, we assume this is less likely (Simunovic et al., 2013)). As in Experiment 2, we did not replicate the significant interaction between time pressure and group membership obtained in Experiments 1 and 3, but again we found a consistent directional trend in the data.

\section{Combined Analysis}

Finally, we took advantage of the similar methods used across the various studies to conduct a combined analysis looking at the effects of group and time manipulation on behavior in the PSG across all four studies $(\mathrm{N}=2894$; excluding the 'unidentified' group condition in Study 4).

We began by conducting two linear mixed effects model using the lme4 package in R, allowing us to compare whether a model allowing for the interaction between group membership and time pressure manipulation was better than one without an interaction (given that in some experiments the interaction effect was significant, and in others it was not). In both models, we included experiment number as a random effect to allow for 
heterogeneity across these experiments. We found that the full model including the interaction indeed had a significantly better fit than a model only with the main effects, $\chi^{2}(1)$ $=6.02, p=.014$. For this reason, we report the results of the full model.

We observed main effects of both group membership, $B=0.72, S E=0.14, Z=5.32, p$ $<0.001$, and time pressure manipulation, $B=0.76, S E=0.18, Z=4.22, p<0.001$; these main effects were qualified by a significant interaction between group condition and time manipulation, $B=-0.67, S E=0.19, Z=-3.56, p<0.001$. Breaking this down, we found that under time pressure participants were equally likely to attack an in-group (26\%) or out-group (27\%) member, $B=-0.06, S E=0.13, Z=-0.43, p=.97$. In contrast, when participants had the chance to deliberate they were significantly more likely to attack an out-group (24\%) than in-group member $(13 \%), B=-0.72, S E=0.14, Z=-5.32, p<.001$. While time pressure made no difference to the incidence of attacking against an out-group member, $B=-0.09, S E=$ $0.15, Z=-0.59, p=.93$, the possibility to deliberate led to significantly fewer attacks against an in-group member than when participants were under time pressure, $B=-0.76, S E=0.18, Z$ $=-4.22, p<.001($ see Figure 6$)$.

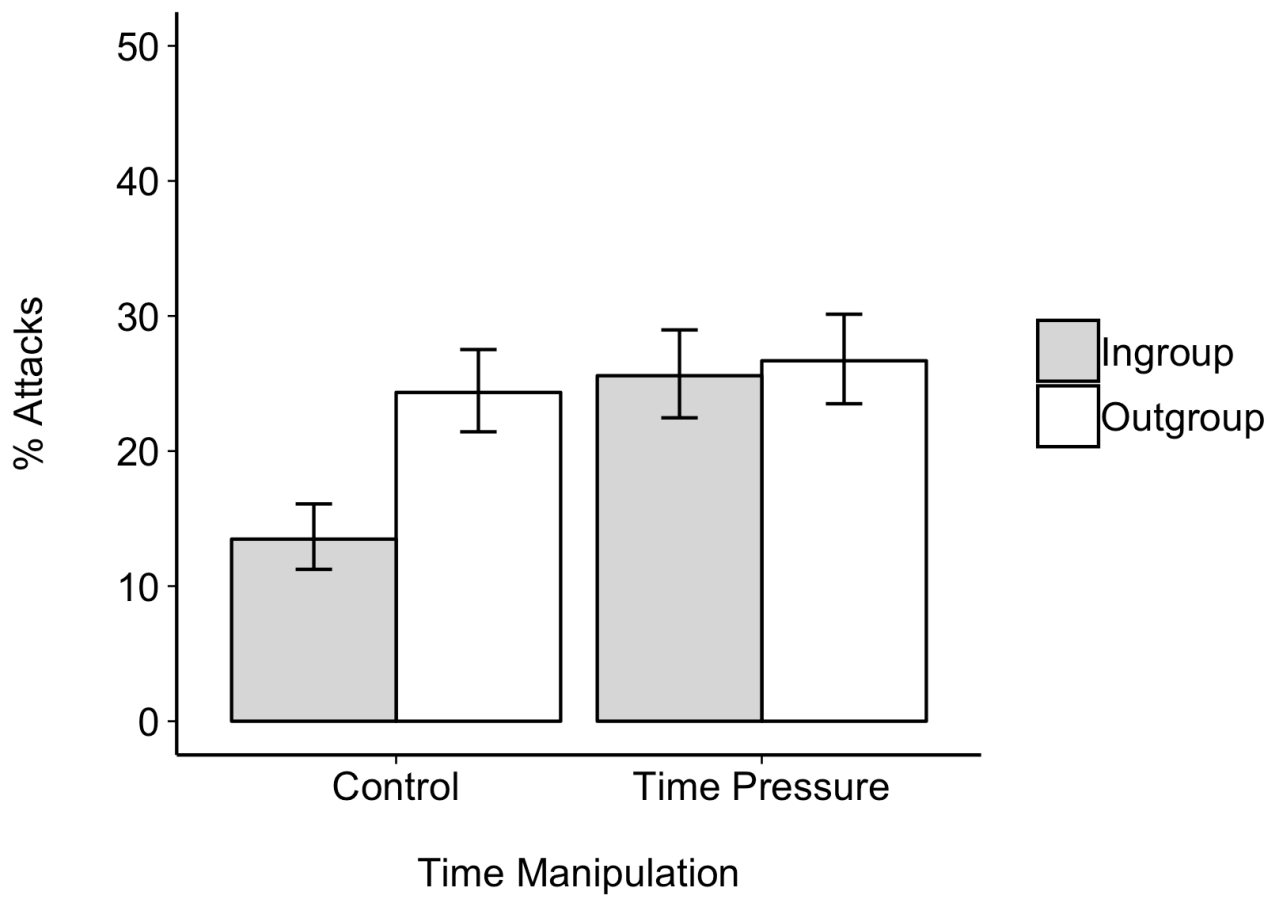


Figure 6. Under time pressure, participants were equally likely to attack an in-group or outgroup member, but when they had the chance to deliberate were significantly more likely to attack an out-group than in-group member. Error bars represent $95 \%$ confidence intervals (Combined Analysis for Experiments 1-4).

\section{General Discussion}

Are humans 'by nature' cooperative? Much contemporary research offers a rosy picture of human social instincts, suggesting that cooperative behavior holds intrinsic value and arises from intuitive, automatic processes, while deliberation tends to undermine it (e.g. Rand, 2016; Zaki \& Mitchell, 2011, 2013). On the other hand, automatic, intuitive processes might also encourage people to be uncooperative or even aggressive by default (e.g. De Dreu et al., 2015; Nelson \& Trainor, 2007) - especially when placed in competitive contexts and/or when interacting with out-group members. Here, we asked when intuitive processes might favor aggression over cooperation, focusing on a kind of defensive aggression known as preemptive strikes: the launching of an attack on another agent to gain a strategic advantage before the other agent can attack you. Modelling this in an economic game, we found that a default of aggression prevailed: under time pressure, participants were more likely to strike. While deliberation consistently and substantially reduced aggression towards ingroup members, and towards individuals of unspecified group membership, it had no consistent effect on aggression towards outgroup members.

In contrast to the conclusions of previous empirical work, our results show that intuition can sometimes favor fear-based, non-cooperative behavior. Yet, the novel context in which we found this pattern is compatible with some existing theoretical treatments. The dominant explanation of intuitive cooperation comes from the social heuristics hypothesis (SHH: Rand, 2016; Rand et al., 2014), which posits that cooperation is typically a successful 
strategy in daily life and so in turn cooperation becomes automatized as a default responses. Applying the logic of the SHH to games designed to measure defensive aggression, we might therefore expect that (1) intuition would favour aggression vs. inaction depending the relative "general" success of these strategies in prior episodes, while (2) deliberation should favour aggression or inaction depending on "situation-specific" beliefs about the likelihood of one's partner attacking. Our findings here provide support for the latter prediction: Presumably, deliberation allows higher rates of striking against out-group members because they are distrusted. Testing the first prediction requires measures of individual differences in default trust or life experience, and this remains an important area for further research. Another promising avenue for research is to integrate the PSG into formal models of the evolution of intuitive and deliberative social behaviour. At least one theoretical model shows that for certain coordination games (like the PSG, but unlike the one-shot PDG), intuitive defection and deliberative cooperation may dominate in an evolutionary equilibrium (Bear, Kagan, \& Rand 2017).

Our four experiments varied in their patterns of statistical significance, but the descriptive pattern of results across all studies was very consistent, and these patterns were strongly confirmed in an analysis aggregating data across our four experiments. Participants were more likely to attack an out-group than in-group member, were more likely to attack their partner under time pressure than when they had the chance to deliberate, and were less likely to attack an in-group than out-group member when they had the chance to deliberate.

Another interesting finding was that overall rates of pre-emptive striking were relatively low, often between $10 \%$ and $30 \%$ across conditions. These specific rates likely reflect the particular payoff structure we implemented. We consider two potential explanations for this pattern of results. First, even though we did use real-world, competitive groups (U.S. political parties), our MTurk participants might still see other MTurkers as a 
superordinate in-group (Almaatouq, Krafft, Dunham, Rand, \& Pentland, 2018), which could reduce the distrust of the outgroup. Second, even though we deliberately used an economic game with actual payoffs, the negative consequences of being attacked (and thus the fear) is still necessarily much lower than in real-world encounters. That is, if a participant chose to cooperate and the other player attacked them, the participant lost out on a few cents in a bonus but was still paid for their time. Compared to real-world cases where the decision to not engage in defensive aggression can be the difference between life and death, it is perhaps unsurprising that we did not observe higher levels of aggression overall.

To summarize, in this paper we presented evidence from four experiments, collectively suggesting that intuition does not just favor cooperation, but also defense-based aggression. We show that relying on intuitive process can encourage pre-emptive strikes, but the potential for deliberation allows for a corrective process that can selectively reduce aggression against ingroup members.

\section{References}

Abbink, K., \& de Haan, T. (2014). Trust on the brink of Armageddon: The first-strike game. European Economic Review, 67, 190-196.

https://doi.org/10.1016/j.euroecorev.2014.01.009

Almaatouq, A., Krafft, P., Dunham, Y., Rand, D. G., \& Pentland, A. (2018). Turkers of the World Unite: Multilevel In-Group Bias Amongst Crowdworkers on Amazon Mechanical Turk (SSRN Scholarly Paper No. ID 3122520). Rochester, NY: Social Science Research Network. Retrieved from https://papers.ssrn.com/abstract=3122520 Böhm, R., Rusch, H., \& Gürerk, Ö. (2016). What makes people go to war? Defensive intentions motivate retaliatory and preemptive intergroup aggression. Evolution and Human Behavior, 37(1), 29-34. https://doi.org/10.1016/j.evolhumbehav.2015.06.005 
Chang, L. W., Krosch, A. R., \& Cikara, M. (2016). Effects of intergroup threat on mind, brain, and behavior. Current Opinion in Psychology, 11, 69-73. https://doi.org/10.1016/j.copsyc.2016.06.004

Cikara, M., \& Van Bavel, J. J. (2014). The neuroscience of intergroup relations: An integrative review. Perspectives on Psychological Science, 9(3), 245-274. https://doi.org/10.1177/1745691614527464

Cottrell, C. A., \& Neuberg, S. L. (2005). Different Emotional Reactions to Different Groups: A Sociofunctional Threat-Based Approach to "Prejudice." Journal of Personality and Social Psychology, 88(5), 770-789. https://doi.org/10.1037/0022-3514.88.5.770

Crockett, M. J., Kurth-Nelson, Z., Siegel, J. Z., Dayan, P., \& Dolan, R. J. (2014). Harm to others outweighs harm to self in moral decision making. Proceedings of the National Academy of Sciences, 111(48), 17320-17325.

https://doi.org/10.1073/pnas.1408988111

Cushman, F., Gray, K., Gaffey, A., \& Mendes, W. B. (2012). Simulating murder: the aversion to harmful action. Emotion (Washington, D.C.), 12(1), 2-7.

https://doi.org/10.1037/a0025071

Cushman, F., Young, L., \& Hauser, M. (2006). The role of conscious reasoning and intuition in moral judgment: Testing three principles of harm. Psychological Science, 17(12), 1082-1089. https://doi.org/10.1111/j.1467-9280.2006.01834.x

Dawes, C. T., Loewen, P. J., Schreiber, D., Simmons, A. N., Flagan, T., McElreath, R., ... Paulus, M. P. (2012). Neural basis of egalitarian behavior. Proceedings of the National Academy of Sciences, 109(17), 6479-6483. https://doi.org/10.1073/pnas.1118653109

De Dreu, C. K. W., \& Gross, J. (Forthcoming). Games of Attack and Defense: Forms and Functions of (Inter)group Conflict. Behavioral and Brain Sciences. 
De Dreu, C. K. W., Scholte, H. S., van Winden, F. A. A. M., \& Ridderinkhof, K. R. (2015). Oxytocin tempers calculated greed but not impulsive defense in predator-prey contests. Social Cognitive and Affective Neuroscience, 10(5), 721-728. https://doi.org/10.1093/scan/nsu109

Dunham, Y., Baron, A. S., \& Banaji, M. R. (2008). The development of implicit intergroup cognition. Trends in Cognitive Sciences, 12(7), 248-253. https://doi.org/10.1016/j.tics.2008.04.006

Everett, J. A. C., Ingbretsen, Z., Cushman, F., \& Cikara, M. (2017). Deliberation erodes cooperative behavior - Even towards competitive out-groups, even when using a control condition, and even when eliminating selection bias. Journal of Experimental Social Psychology, 73, 76-81. https://doi.org/10.1016/j.jesp.2017.06.014

Greene, J. D. (2008). The secret joke of Kant's soul. In Moral psychology, Vol 3: The neuroscience of morality: Emotion, brain disorders, and development (pp. 35-80). Cambridge, MA, US: MIT Press.

Halevy, N. (2017). Preemptive strikes: Fear, hope, and defensive aggression. Journal of Personality and Social Psychology, 112(2), 224-237. https://doi.org/10.1037/pspi0000077

Harsanyi, J. C., \& Selten, R. (1988). A General Theory of Equilibrium Selection in Games (Vol. 1). The MIT Press. Retrieved from https://ideas.repec.org/b/mtp/titles/0262582384.html

Insko, C. A., \& Schopler, J. (1998). Differential distrust of groups and individuals. In C. Sedikides, J. Schopler, \& C. Asko (Eds.), Intergroup cognition and intergroup behavior (pp. 75-107). Mahwah, NJ, US: Lawrence Erlbaum Associates Publishers. 
Levine, E. E., Barasch, A., Rand, D., Berman, J. Z., \& Small, D. A. (2018). Signaling emotion and reason in cooperation. Journal of Experimental Psychology: General, 147(5), 702-719. https://doi.org/10.1037/xge0000399

Lotz, S. (2015). Spontaneous Giving under Structural Inequality: Intuition Promotes Cooperation in Asymmetric Social Dilemmas. PLOS ONE, 10(7), e0131562. https://doi.org/10.1371/journal.pone.0131562

Martinez, J. E., Feldman, L. A., Feldman, M. J., \& Cikara, M. (Under Review). Criminality racializes, achievement homogenizes: Narratives shape cognitive representations of immigrants.

Mifune, N., Hashimoto, H., \& Yamagishi, T. (2010). Altruism toward in-group members as a reputation mechanism. Evolution and Human Behavior, 31(2), 109-117. https://doi.org/10.1016/j.evolhumbehav.2009.09.004

Mifune, N., Hizen, Y., Kamijo, Y., \& Okano, Y. (2016). Preemptive Striking in Individual and Group Conflict. PLOS ONE, 11(5), e0154859. https://doi.org/10.1371/journal.pone.0154859

Nelson, R. J., \& Trainor, B. C. (2007). Neural mechanisms of aggression. Nature Reviews. Neuroscience, 8(7), 536-546. https://doi.org/10.1038/nrn2174

Pemberton, M. B., Insko, C. A., \& Schopler, J. (1996). Memory for and experience of differential competitive behavior of individuals and groups. Journal of Personality and Social Psychology, 71(5), 953. https://doi.org/10.1037/0022-3514.71.5.953

Rand, D. G. (2016). Cooperation, fast and slow: Meta-analytic evidence for a theory of social heuristics and self-interested deliberation. Psychological Science, 27(9), 1192-1206. https://doi.org/10.1177/0956797616654455

Rand, D. G., Greene, J. D., \& Nowak, M. A. (2012). Spontaneous giving and calculated greed. Nature, 489(7416), 427-430. https://doi.org/10.1038/nature11467 
Rand, D. G., Peysakhovich, A., Kraft-Todd, G. T., Newman, G. E., Wurzbacher, O., Nowak, M. A., \& Greene, J. D. (2014). Social heuristics shape intuitive cooperation. Nature Communications, 5, 3677. https://doi.org/10.1038/ncomms4677

Riek, B. M., Mania, E. W., \& Gaertner, S. L. (2006). Intergroup Threat and Outgroup Attitudes: A Meta-Analytic Review. Personality and Social Psychology Review, 10(4), 336-353. https://doi.org/10.1207/s15327957pspr1004_4

Sanfey, A. G., Rilling, J. K., Aronson, J. A., Nystrom, L. E., \& Cohen, J. D. (2003). The Neural Basis of Economic Decision-Making in the Ultimatum Game. Science, 300(5626), 1755-1758. https://doi.org/10.1126/science.1082976

Schelling, T. C. (1960). The reciprocal fear of surprise attack. In The Strategy of Conflict (pp. 207-229). Cambridge, MA: Harvard University Press.

Sherif, M. (1966). In common predicament: Social psychology of intergroup conflict and cooperation. Boston, MA: Houghton Mifflin.

Simunovic, D., Mifune, N., \& Yamagishi, T. (2013). Preemptive strike: An experimental study of fear-based aggression. Journal of Experimental Social Psychology, 49(6), 1120-1123. https://doi.org/10.1016/j.jesp.2013.08.003

Stephan, W. G., Renfro, C., \& Davis, M. D. (2008). The role of threat in intergroup relations. Improving Intergroup Relations: Building on the Legacy of Thomas F. Pettigrew, 5572.

Tricomi, E., Rangel, A., Camerer, C. F., \& O’Doherty, J. P. (2010). Neural evidence for inequality-averse social preferences. Nature, 463(7284), 1089-1091. https://doi.org/10.1038/nature08785

Zaki, J., López, G., \& Mitchell, J. P. (2014). Activity in ventromedial prefrontal cortex covaries with revealed social preferences: evidence for person-invariant value. Social 
Cognitive and Affective Neuroscience, 9(4), 464-469.

https://doi.org/10.1093/scan/nst005

Zaki, J., \& Mitchell, J. P. (2011). Equitable decision making is associated with neural markers of intrinsic value. Proceedings of the National Academy of Sciences, 108(49), 1976119766. https://doi.org/10.1073/pnas. 1112324108

Zaki, J., \& Mitchell, J. P. (2013). Intuitive prosociality. Current Directions in Psychological Science, 22(6), 466-470. https://doi.org/10.1177/0963721413492764 